\title{
Transient HIV Reservoirs and Interleukin-6 Increase After Anti-Programmed Death-I Antibody Infusion in HIV Patients with Lung Cancer
}

\author{
Xiaorong Peng \\ Ran Tao \\ Ying Chen \\ Junwei Su \\ Ying Huang \\ Biao Zhu
}

The Department of Infectious Diseases, State Key Laboratory for Diagnosis and Treatment of Infectious Diseases, Collaborative Innovation Center for Diagnosis and Treatment of Infectious Diseases, National Clinical Research Center for Infectious Diseases, The First Affiliated Hospital, School of Medicine, Zhejiang University, Hangzhou, 310006 , People's Republic of China
Correspondence: Biao Zhu

Email zhubiaol207@zju.edu.cn
Background: The purpose of this study is to examine the dynamic effects of antiprogrammed death (PD)-1 antibody treatment on HIV reservoirs and inflammatory cytokines in patients with HIV infections who were diagnosed with non-small cell lung cancer (NSCLC).

Methods: This is a clinical trial in which three HIV patients with NSCLC were administered 14 infusions of anti-PD-1 antibody at 21-day intervals. Blood samples were collected from the participants before each infusion $(0 \mathrm{~h})$, and at $2 \mathrm{~h}, 24 \mathrm{~h}$, and 7 days after each infusion of anti-PD-1 monoclonal antibody. The levels of cell-associated HIV RNA (CAHIV-RNA), HIV DNA, and inflammatory cytokines (including interferon [IFN]- $\alpha$, IFN- $\gamma$, tumor necrosis factor [TNF]- $\alpha$, interleukin [IL]-2, IL-4, IL-6, IL-10, and IL-17A) were assessed at each timepoint.

Results: A significant increase in CA-HIV-RNA $(\mathrm{P}=0.049)$ and HIV DNA $(\mathrm{P}=0.042)$ was observed $24 \mathrm{~h}$ after each infusion of anti-PD-1 monoclonal antibody. The $Z$-score for IL-6 increased from $-0.46 \pm 0.53$ to $0.28 \pm 0.78$ at $24 \mathrm{~h}$ after infusion $(\mathrm{P}=0.02)$, and further increased to $0.61 \pm 1.0$ at 7 days after each infusion $(\mathrm{P}=0.007)$. A significant correlation was observed between IL-6 and HIV DNA $(\mathrm{P}=0.02)$.

Conclusion: The CA-HIV-RNA, HIV DNA, and IL-6 levels significantly increased after infusion of anti-PD-1 monoclonal antibody in the three HIV-infected patients with NSCLC. These results support an early transient effect of anti-PD-1 monoclonal antibody infusion on HIV reservoirs. However, the long-term effect needs to be investigated in a larger cohort with a longer follow-up period.

Keywords: anti-PD-1 antibody, HIV, CA-HIV-RNA, HIV DNA, IL-6

\section{Introduction}

Anti-programmed death-1 (PD-1) antibody, which is an immune checkpoint inhibitor, has become the new standard treatment agent for advanced non-small cell lung cancer (NSCLC). ${ }^{1}$ Recent research has suggested that it could also be a therapeutic option in human immunodeficiency virus (HIV) infections. ${ }^{2} \mathrm{~A}$ few case reports on anti-PD-1 antibody treatment of HIV-infected patients with NSCLC have shown encouraging results, but the antiviral effects of anti-PD-1 antibody are unclear. $^{3}$ Guihot et al reported a drastic and persistent decrease from 369 at D0 to 30 copies $/ 10^{6}$ cells at D120 in the cell-associated HIV DNA in a patient treated with anti-PD-1 monoantibody for lung cancer. ${ }^{4}$ However, Le Garff et al described a similar case with little or no decrease in the cell-associated HIV DNA (116 copies 
at D0, 213 copies $/ 10^{6}$ cells at D30). ${ }^{5}$ Given these contradictory findings, the effects of administering anti-PD-1 antibody on the HIV reservoir need further research.

Chronic HIV infection is associated with a persistent inflammatory response marked by the production of excess amounts of a number of pro-inflammatory cytokines, including interferon (IFN)- $\alpha$, IFN- $\gamma$, tumor necrosis factor (TNF)- $\alpha$, interleukin (IL)-6, IL-8, and IL-15. The levels of these markers decrease with antiretroviral therapy (ART), but they are still higher than those in individuals who do not have HIV infection. ${ }^{7}$ When the PD-1/PD-L1 axis is blocked, the level of inflammatory cytokines usually increases as a result of the activation of immune cells, including $\mathrm{T}$ cells, natural killer cells, and macrophages. ${ }^{6}$ This cytokine release is commonly associated with elevated levels of IL-6, IFN- $\gamma$, and other cytokines. Therefore, further studies are warranted to determine the dynamic effects of anti-PD- 1 antibody treatment on pro-inflammatory cytokine levels in HIV infections.

Previous studies have shown that anti-PD-1 therapy is safe and very effective in some HIV-positive patients with lung cancer. ${ }^{3}$ However, their findings are limited by the small number of patients and the administration of only a single dose of anti-PD-1 antibody. Therefore, a pilot study on three HIV-infected patients with lung cancer was conducted to examine the clinical effectiveness of anti-PD-1 antibody therapy and its effect on the HIV reservoir and inflammatory cytokines.

\section{Methods}

\section{Participants}

This pilot study was conducted in compliance with the Declaration of Helsinki and approved by the institutional review board of the First Affiliated Hospital, School of Medicine, Zhejiang University (Reference Number: 2020265). Written informed consent was provided by the patients for the use of their clinical information and the publishing of any accompanying images. This study was registered in the Chinese Clinical Trial Registry (Registration Number: ChiCTR1900023184).

The inclusion criteria included HIV infection, age between 18 and 65 years, a definitive diagnosis of cancer, and willingness to undergo therapy with anti-PD-1 monoclonal antibody. Participants with severe comorbidities and acute conditions were excluded. A total of three participants were enrolled and hospitalized in the First Affiliated Hospital, School of Medicine, Zhejiang University.

\section{Sample Collection}

Blood samples were collected from the participants at the following time points: before each infusion $(0 \mathrm{~h})$, and $2 \mathrm{~h}$, $24 \mathrm{~h}$, and $7 \mathrm{~d}$ after each infusion of anti-PD-1 monoclonal antibody. Plasma was separated by centrifugation and stored at $-80^{\circ} \mathrm{C}$ until use. Peripheral blood mononuclear cells (PBMCs) were isolated in a Ficoll-Hypaque gradient by density gradient centrifugation, and two million PBMCs were stored in liquid nitrogen. HIV plasma viral load was measured using the Abbott Real-Time HIV-1 assay (Abbott Laboratories, Abbott Park, Illinois, USA) (lower limit of detection $=20$ copies $/ \mathrm{mL}$ ). CD4+ and CD8 + T-cell counts were measured using four-color flow cytometry. The serum IL-2, IL-4, IL-6, IL-10, TNF- $\alpha$, IL-17A, and IFN- $\gamma$ levels were measured by the commercially enzyme-linked immunosorbent Human Platinum ELISA kits (eBioscience, North America).

\section{HIV Reservoir Quantification}

Cell-associated HIV RNA (CA-HIV-RNA) in PBMCs was measured with HIV-1 RT-PCR Assay V2 (Qiagen, Hilden, Germany). HIV DNA in PBMCs was amplified and quantified for the LTR gene using a fluorescence-based, realtime SUPBIO HIV Quantitative Detection Kit (SUPBIO, Guangzhou, China).

\section{Statistical Analysis}

Data were analyzed for statistical significance using GraphPad Prism version 5.02 (GraphPad Software Inc., San Diego, CA). P $<0.05$ was considered to indicate statistical significance. In order to analyze the time variation of parameters while accounting for inter-cycle variability, the individual raw data were converted to $Z$ scores as previously described. ${ }^{8} Z$-scores were calculated as follows, by normalizing each datapoint to the mean of the values for the four timepoints: (datapoint value - mean value)/standard deviation. Multiple comparisons were made between timepoints using non-parametric Friedman test. Spearman test was used to test the correlation between IL-6 levels and HIV reservoir markers.

\section{Results}

\section{Patient Characteristics}

A total of 3 participants who initiated antiretroviral therapy during chronic HIV infection and diagnosed with NSCLC were analyzed. The median age was 44 years (range: $36-55$ ) and $100 \%$ were male. At the time of chemotherapy, median 
CD4 count was $601 / \mathrm{mm}^{3}$ (243-864). One participant had an HIV viral load of 73 copies/mL. Overall change in plasma HIV RNA was from 20 copies/mL (lower limit of detection) to 50.3 copies $/ \mathrm{mL}$ during the follow-up. The rest two participants maintained virologic suppression during the chemotherapy.

The clinical characteristics of these three participants are presented in Table 1.

\section{Variations in the HIV Reservoir After Each Infusion of Anti-PD-I Monoclonal Antibody}

The Z-score results showed that the CA-HIV-RNA ( $\mathrm{P}=$ $0.049)$ and HIV DNA $(\mathrm{P}=0.042)$ levels were significantly increased $24 \mathrm{~h}$ after infusion of anti-PD-1 monoclonal antibody and were also correlated (Figure 1).

\section{Variations in Cytokine Levels After Each Infusion of Anti-PD-I Monoclonal \\ Antibody}

The levels of IL-2 ( $\mathrm{P}=0.41)$, IL-4 ( $\mathrm{P}=0.11)$, IL-10 ( $\mathrm{P}=0.19)$, TNF- $\alpha(\mathrm{P}=0.12)$, IL17 $\alpha(\mathrm{P}=0.09)$, and IFN- $\gamma(\mathrm{P}=0.14)$ did not vary significantly after the anti-PD-1 monoclonal antibody infusions (Figures S1 and S2). However, the IL-6 levels showed significant variations $(\mathrm{P}=0.002)$. Before infusion of anti-PD-1 monoclonal antibody, the $Z$-score for IL-6 levels was $-0.46 \pm 0.53$, and it increased to $0.28 \pm 0.78$ at $24 \mathrm{~h}$ after infusion of anti-PD-1 monoclonal antibody $(\mathrm{P}=0.02)$. The $Z$-score of the IL-6 levels further increased to $0.61 \pm 1.0$ at 7 days after infusion of anti-PD-1 monoclonal antibody $(\mathrm{P}=0.007)$ (Figure $1 \mathrm{G}-\mathrm{I})$.

\section{Association Between HIV Reservoirs and IL-6 Levels}

As previous studies have shown that IL-6 is a major factor in the persistence of HIV reservoirs, we sought to determine whether the serum concentrations of IL-6 were correlated with the CA-HIV-RNA and HIV DNA levels. A significant correlation was found between IL6 and HIV DNA $(\mathrm{P}=0.02)$ (Figure $1 \mathrm{~K})$, but no correlation was observed between IL-6 and CA-HIV-RNA $(P=0.06)($ Figure $1 \mathrm{~J})$.

\section{Discussion}

In this study, anti-PD-1 monoclonal antibody infusion was found to be safe and well tolerated in three individuals with

\begin{tabular}{|c|c|c|c|}
\hline 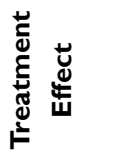 & 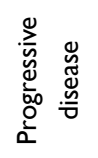 & 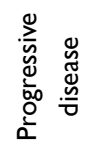 & 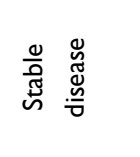 \\
\hline 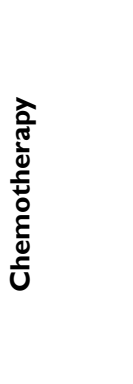 & 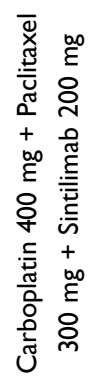 & 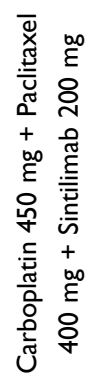 & 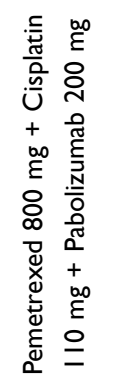 \\
\hline 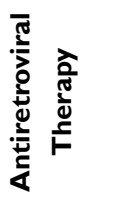 & \multicolumn{3}{|c|}{ 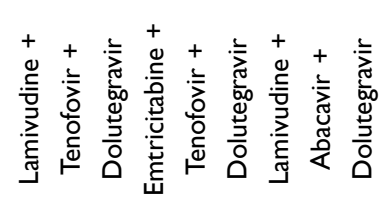 } \\
\hline 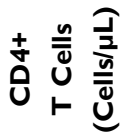 & $\stackrel{m}{\sim}$ & $\overline{8}$ & ర్ \\
\hline 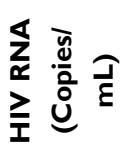 & $\Re$ & 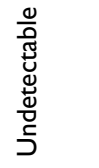 & 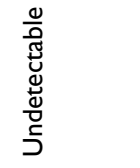 \\
\hline 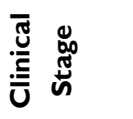 & $\stackrel{\infty}{\geq}$ & $\stackrel{\varrho}{\cong}$ & $\leqq$ \\
\hline 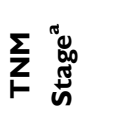 & 豙 & 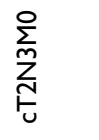 & 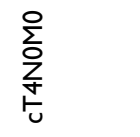 \\
\hline 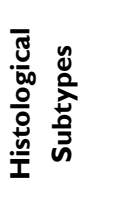 & 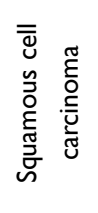 & 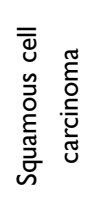 & 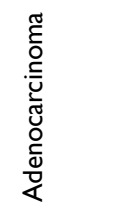 \\
\hline 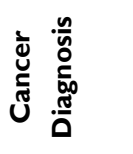 & $\frac{\stackrel{n}{\circ}}{\stackrel{\alpha}{\circ}}$ & 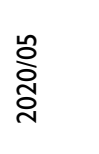 & $\frac{\stackrel{0}{N}}{\stackrel{N}{人}}$ \\
\hline 主 & $\frac{n}{\bar{N}}$ & ఝ్రి & $\stackrel{\nabla}{\bar{N}}$ \\
\hline 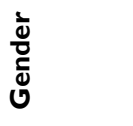 & $\frac{\frac{0}{\pi}}{\frac{\pi}{\Sigma}}$ & $\frac{\frac{\omega}{\pi N}}{\sum}$ & $\frac{\frac{\omega}{\pi N}}{\sum}$ \\
\hline$\stackrel{8}{8}$ & J & นn & $\stackrel{\circ}{m}$ \\
\hline$\underline{\underline{0}}$ & - & N & $m$ \\
\hline
\end{tabular}



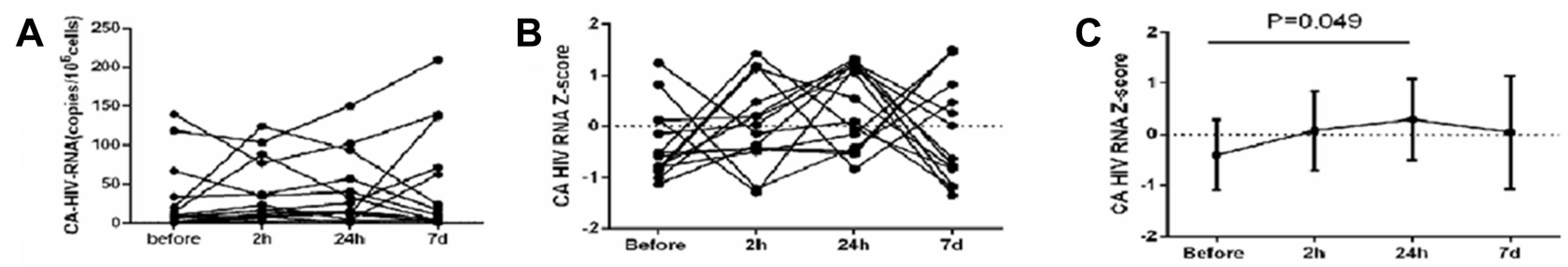

D

E

F
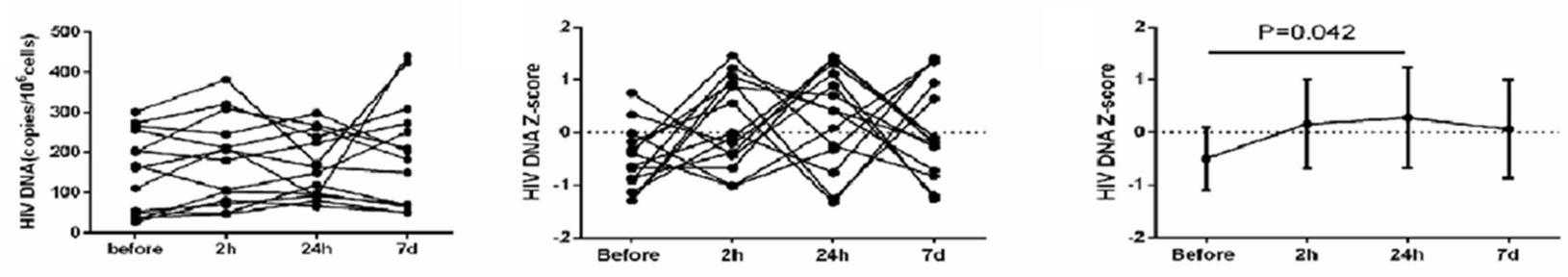

G

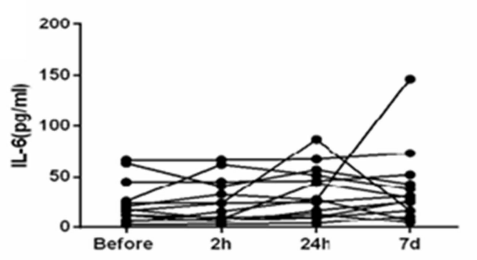

H

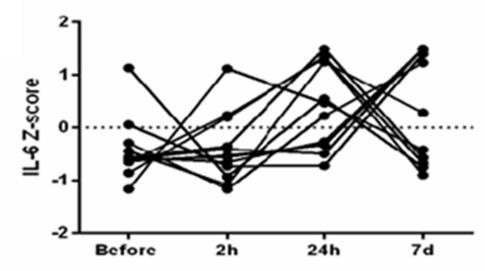

I
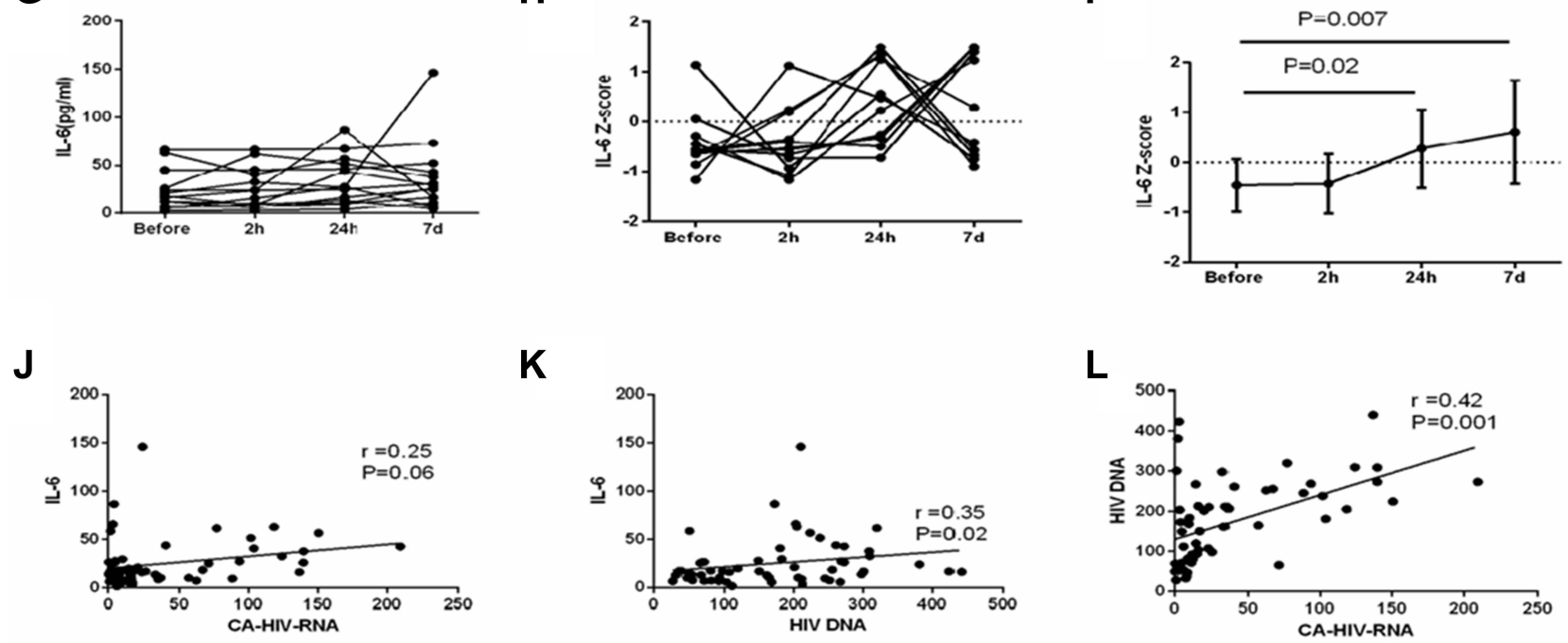

K
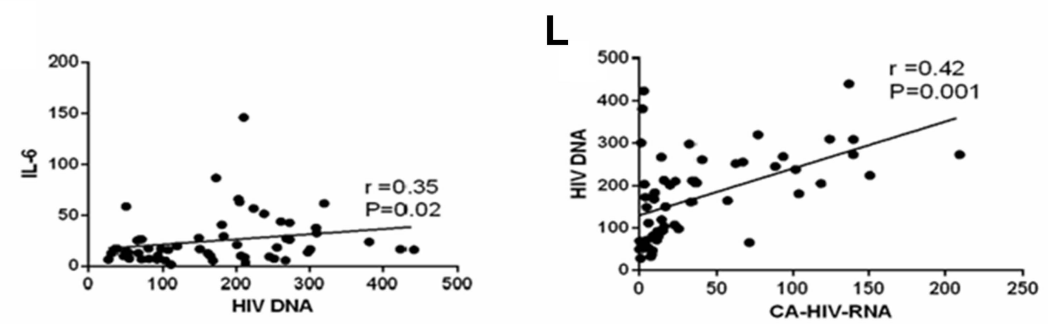

Figure I Effect of anti-PD-I monoclonal antibody infusion on cell-associated HIV RNA, HIV DNA, and IL-6. Variations in cell-associated HIV RNA (CA-HIV-RNA) (A-C), HIV DNA (D-F), and IL-6 after each infusion of anti-PD-I monoclonal antibody (G-I). Mean \pm standard deviation values of the Z-score are shown in panels C, F, and I. A significant correlation was found between IL-6 and HIV DNA (K), but no correlation was observed between IL-6 and CA-HIV-RNA (J). The CA-HIV-RNA levels were also correlated with the HIV DNA levels in HIV-infected patients (L).

chronic ART-suppressed HIV-1 infection and lung cancer. Our data show that the CA-HIV-RNA, HIV DNA, and IL-6 levels transiently increased after anti-PD-1 monoclonal antibody treatment.

In the present study, the levels of CA-HIV-RNA and HIV DNA, which are markers of HIV persistence, were significantly increased from the baseline to $24 \mathrm{~h}$ after infusion of anti-PD-1 monoclonal antibody. One previous study has shown a two-fold increase in cell-associated HIV DNA levels on day 30 after administration of anti-PD-1 monoclonal antibody. ${ }^{5}$ Another case report documented that the administration of anti-PD-1 to an HIV-infected individual on ART resulted in a significant increase in CA-HIV-RNA levels in CD4 lymphocyte, without significant changes in HIV DNA or plasma HIV RNA levels on day 7 after treatment. ${ }^{9}$ These differences could be explained by several reasons. Firstly, different cells (PMBC or CD4+ T cells) were used for HIV reservoir measurements. Secondly, different methods were conducted to quantification HIV reservoir (CA-HIV-RNA or HIV DNA). Thirdly, different anti-PD-1 monoclonal antibodies (nivolumab, pabolizumab or sintilimab) were administered.

HIV persistence, which is one of the barriers to curing the infection, is enriched in PD-1-positive CD4 
lymphocyte, ${ }^{10}$ which are reactivated by anti-PD-1 antibody. ${ }^{11}$ Examination of CD4 lymphocyte from HIVinfected individuals has shown that PD-1 blockade significantly enhanced HIV production and increased the levels of cytokine production without increasing T-cell activation. ${ }^{11}$ Accordingly, in the present study, IL-6 levels increased from the baseline to $24 \mathrm{~h}$ and 7 days after infusion of anti-PD-1 monoclonal antibody. Our data also showed that IL-6 was correlated with HIV DNA levels. IL-6, as an inflammatory stimulus, can also cause activation of resting $\mathrm{T}$ cells, and theoretically, activated CD4 lymphocyte may contribute to HIV persistence by continuously providing a pool of cellular targets for the virus to infect. $^{7}$ On the other hand, anti-PD-1 antibody could "unshield" HIV-infected cells and enhance the effector function of HIV-specific CD8 lymphocyte at tissue sites. ${ }^{12}$ However, our study showed evidence of an early transient effect of anti-PD-1 monoclonal antibody infusion on HIV reservoir. The long-term effect need to be investigated in a larger cohort with a longer follow-up period.

Additionally, it is known that IL-6 has immunosuppressive functions and may drive a myeloid compartment that contributes to innate treatment resistance, for example, the accumulation of T-cell-suppressing neutrophils. ${ }^{13}$ For example, Tsukamoto et al showed that increased IL-6 levels were associated with decreased efficacy of PD-1 blockade in patients with melanoma, and that IL-6 blockade augments PD-L1 expression in tumor cells. ${ }^{14}$ In contrast, in a study of 10 patients with NSCLC, the group with elevated IL-6 levels in response to immunotherapy showed higher response rates than the group that did not have elevated IL-6 levels. ${ }^{15}$ Thus, whether IL-6 elevation is beneficial for the effects of PD-1 is still controversial.

The findings of this study are limited because of the small sample size. Additionally, longer follow-up is required to determine the long-term effect of anti-PD-1 antibody treatment on the HIV reservoir. Nonetheless, the findings contribute to the sparse information that is currently available.

In summary, our study demonstrated an early transient increase in CA-HIV-RNA, HIV DNA, and IL-6 after infusion of anti-PD-1 monoclonal antibody in HIV-infected patients with NSCLC. However, the long-term effects need to be assessed in a larger cohort with a longer followup period.

\section{Data Sharing Statement}

The datasets used in this study are available from the corresponding author on reasonable request.

\section{Ethics Approval}

This study was approved by the institutional review board of the First Affiliated Hospital, School of Medicine, Zhejiang University (Reference Number: 2020265).

\section{Acknowledgments}

This work was supported by the National Key R\&D Program of China (No.2021YFC23019002021YFC2301901) and Major Program of National Natural Science Foundation of China (No. 2017ZX10202102-002-002).

\section{Disclosure}

The authors declare no competing interests.

\section{References}

1. Ettinger DS, Wood DE, Aggarwal C, et al. NCCN guidelines insights: non-small cell lung cancer, Version 1.2020. J Natl Compr Canc Netw. 2019;17:1464-1472. doi:10.6004/jnccn.2019.0059

2. Wykes MN, Lewin SR. Immune checkpoint blockade in infectious diseases. Nat Rev Immunol. 2018;18:91-104. doi:10.1038/nri.2017.112

3. Ostios-Garcia L, Faig J, Leonardi GC, et al. Safety and efficacy of PD-1 inhibitors among HIV-positive patients with non-small cell lung cancer. J Thorac Oncol. 2018;13:1037-1042. doi:10.1016/j.jtho.2018.03.031

4. Guihot A, Marcelin AG, Massiani MA, et al. Drastic decrease of the HIV reservoir in a patient treated with nivolumab for lung cancer. Ann Oncol. 2018;29:517-518. doi:10.1093/annonc/mdx696

5. Le Garff G, Samri A, Lambert-Niclot S, et al. Transient HIV-specific $T$ cells increase and inflammation in an HIV-infected patient treated with nivolumab. AIDS. 2017;31:1048-1051. doi:10.1097/ QAD.0000000000001429

6. Costantini A, Julie C, Dumenil C, et al. Predictive role of plasmatic biomarkers in advanced non-small cell lung cancer treated by nivolumab. Oncoimmunology. 2018;7:e1452581. doi:10.1080/ 2162402X.2018.1452581

7. Klatt NR, Chomont N, Douek DC, Deeks SG. Immune activation and HIV persistence: implications for curative approaches to HIV infection. Immunol Rev. 2013;254:326-342. doi:10.1111/imr.12065

8. Koshy A, Cuesta M, Boudreau P, Cermakian N, Boivin DB. Disruption of central and peripheral circadian clocks in police officers working at night. FASEB J. 2019;33:6789-6800. doi:10.1096/fj.201801889R

9. Evans VA, van der Sluis RM, Solomon A, et al. Programmed cell death-1 contributes to the establishment and maintenance of HIV-1 latency. AIDS. 2018;32:1491-1497. doi:10.1097/QAD.0000000000001849

10. Llewellyn GN, Seclen E, Wietgrefe S, et al. Humanized mouse model of HIV-1 latency with enrichment of latent virus in PD-1(+) and TIGIT(+) CD4 T cells. $J$ Virol. 2019;93. doi:10.1128/JVI.02086-18

11. Fromentin R, DaFonseca S, Costiniuk CT, et al. PD-1 blockade potentiates HIV latency reversal ex vivo in CD4(+) T cells from ART-suppressed individuals. Nat Commun. 2019;10:814. doi:10.1038/s41467-019-08798-7

12. Chen H, Moussa M, Catalfamo M. The role of immunomodulatory receptors in the pathogenesis of HIV infection: a therapeutic opportunity for HIV cure? Front Immunol. 2020;11:1223. doi:10.3389/ fimmu.2020.01223

13. Rossi JF, Lu ZY, Massart C, Levon K. Dynamic immune/inflammation precision medicine: the good and the bad inflammation in infection and cancer. Front Immunol. 2021;12:595722. doi:10.3389/ fimmu.2021.595722 
14. Tsukamoto H, Fujieda K, Miyashita A, et al. Combined blockade of IL6 and PD-1/PD-L1 signaling abrogates mutual regulation of their immunosuppressive effects in the tumor microenvironment. Cancer Res. 2018;78:5011-5022. doi:10.1158/0008-5472.CAN-18-0118
15. Ozawa Y, Amano Y, Kanata K, et al. Impact of early inflammatory cytokine elevation after commencement of PD-1 inhibitors to predict efficacy in patients with non-small cell lung cancer. Med Oncol. 2019;36:33. doi:10.1007/s12032-019-1255-3

\section{Publish your work in this journal}

The Journal of Inflammation Research is an international, peerreviewed open-access journal that welcomes laboratory and clinical findings on the molecular basis, cell biology and pharmacology of inflammation including original research, reviews, symposium reports, hypothesis formation and commentaries on: acute/chronic inflammation; mediators of inflammation; cellular processes; molecular mechanisms; pharmacology and novel anti-inflammatory drugs; clinical conditions involving inflammation. The manuscript management system is completely online and includes a very quick and fair peerreview system. Visit http://www.dovepress.com/testimonials.php to read real quotes from published authors. 